Scientific Journal of Hamadan Nursing \& Midwifery Faculty - ISSN 2008-2819

\title{
Investigation of Factors Affecting on Relapse of Addiction in Substance Abuse Patients Referred to Narcotics Anonymous Population
}

\author{
Mohammad Ahmadpanah', Mohammad Haghighi', Mohammad Behfar², Abbas Moradi \\ Marzieh Nazaribadie ${ }^{4}$
}

1. Research Center for Behavioral Disorders and Substance Abuse, Hamadan University of Medical Sciences, Hamadan, Iran

2. MD, Research Center for Behavioral Disorders and Substance Abuse, Hamadan University of Medical Sciences, Hamadan, Iran

3. Instructor, Department of Social Medicine, Faculty of Medicine, Hamadan University of Medical Sciences, Hamadan, Iran

4. PhD Student, Research Center for Behavioral Disorders and Substance Abuse, Hamadan University of Medical Sciences, Hamadan, Iran

\begin{tabular}{ll}
\hline \multicolumn{2}{c}{ Article Info } \\
\hline & \\
Received: & $2017 / 05 / 22$ \\
Accepted: & $2017 / 06 / 17$ \\
Published Online & $2017 / 07 / 17$
\end{tabular}

DOI:

10.30699/sjhnmf.26.4.247

Original Article

Use your device to scan and read the article online

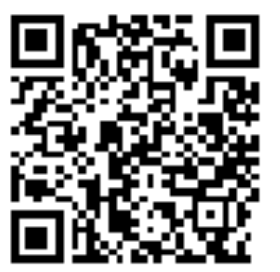

\section{Abstract}

Introduction: The aim of this study was to investigate factors affecting on relapse of addiction in substance abuse patients referred to Malayer Narcotics Anonymous Population.

Methods:This cross-sectional study was performed on 340 substance abuse patients from Narcotics Anonymous population of Malayer recruited with a simple sampling method, in 2014. The standard questionnaire was used for data collection including demographic variables and causes of relapse via self-report method. Data analysis was performed using SPSS18 by Pearson Correlation test.

Results: Age of respondents ranged from 13 to 71 year; with a mean age of 36.47 years $(\mathrm{SD}=11.4)$. According to the results, 58.2 percent of the respondents had 1-3 relapse, 30.9 percent $4-10$ relapse and 10.9 percent more than 10 relapse. The participants reported their viewpoints regarding the other factors as follows: Among individual factors; enjoyment and fun (\%46.2), and among familial factors, poor parental relationships $(\% 25.6)$ had been reported by the addicts. With regard to the social factors, the existence of addicts' friends (52.4\%), and related to the economic factors, lack of job was the major element with (34.1\%). Considering cultural factors, recreational use of drugs and loss of function obscenity (\%55) were leading factors mentioned by the addicts. Findings indicated that all factors related with relapse (personal factors, family factors related to spouse, social factors, economic factors and cultural factors) are significant positive correlation with each other at level in $05 / 0$ and $01 / 0$ respectively.

Conclusion: According to the findings, exploring these correlates may predict the likelihood of relapse among drug users.

Keywords: Relapse; Substance abuse; Narcotics anonymous
Corresponding Information
Marzieh Nazaribadie, PhD Student, Research Center for Behavioral Disorders and Substance Abuse, Hamadan University of Medical Sciences, Hamadan, Iran. Email: mnazaribadie@gmail.com

Copyright (C) 2018, Sci J Hamadan Nurs Midwifery Fac. This is an open-access article distributed under the terms of the Creative Commons Attribution-noncommercial 4.0 International License which permits copy and redistribute the material just in noncommercial usages, provided the original work is properly cited.

How to Cite This Article:

Ahmadpanah M, Haghighi M, Behfar M, Moradi A, Nazaribadie M. Investigation of Factors Affecting on Relapse of Addiction in Substance Abuse Patients Referred to Narcotics Anonymous Population . Sci J Hamadan Nurs Midwifery Fac. 2018; 26 (4) :247-256 
مجله علمى دانشكدهُ يرستارى و مامايى همدان - شايا الكترونيك: 19

مقاله پزوهشى

بررسى عوامل مؤثر بر عود در افراد وابسته به سوءمصرف موادمخدر؛ مطالعهُ موردى مر اجعهكنندَّان به انجمن

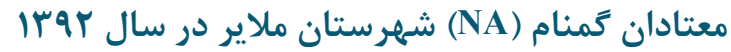

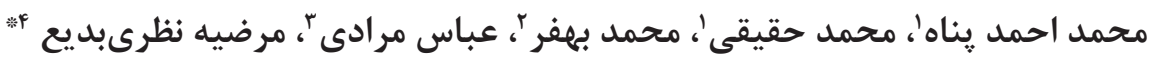

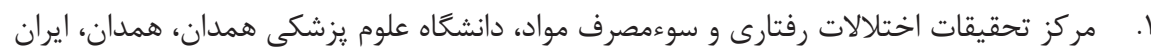

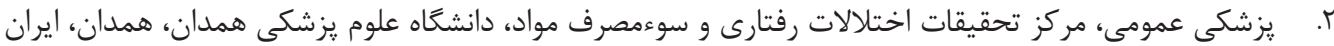

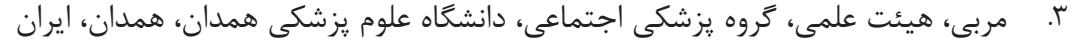

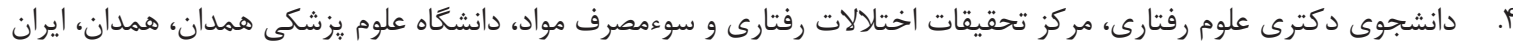

\begin{tabular}{|c|c|}
\hline جكيده & اطلاعات مقاله \\
\hline 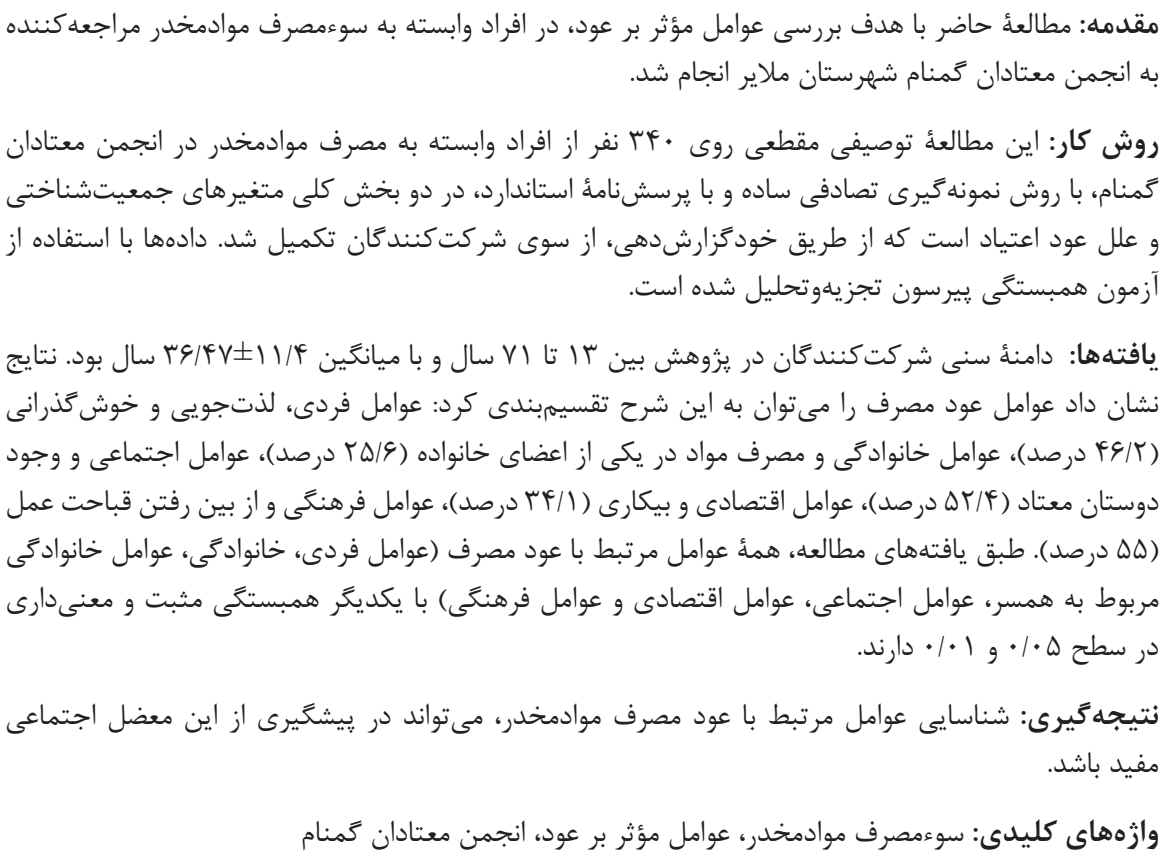 & 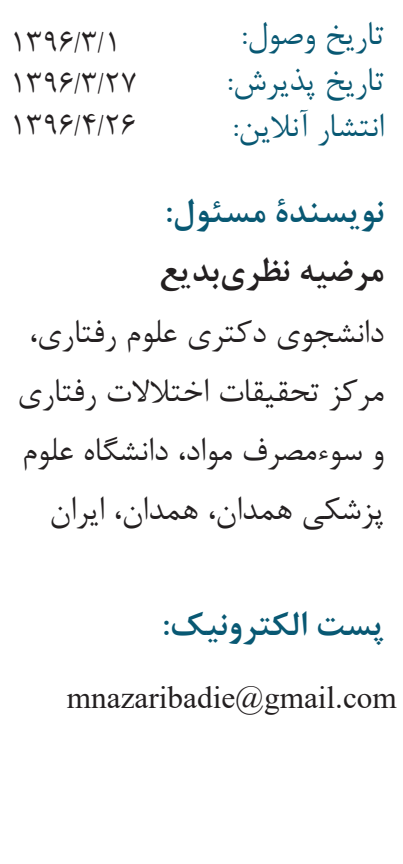 \\
\hline
\end{tabular}

مقلمهـ

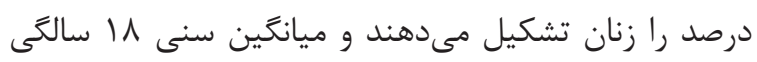

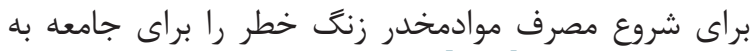

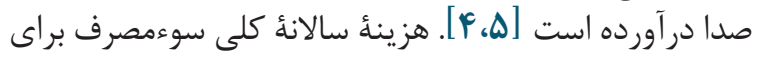

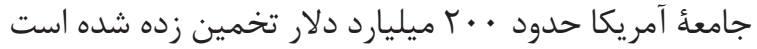

از سوى ديخر يكى از جنبههاى مهرم سوءمصرف كه

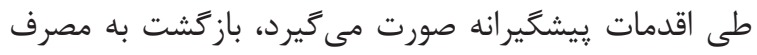

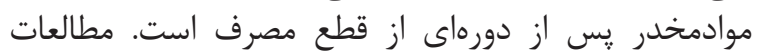

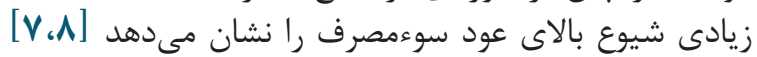

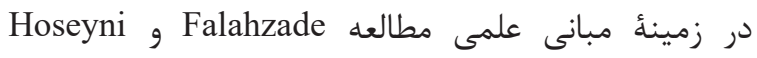

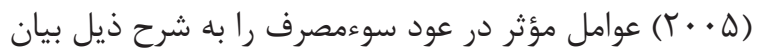

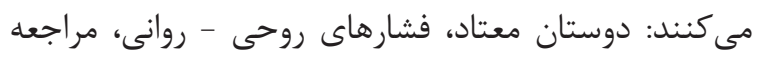

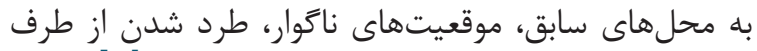

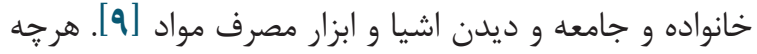

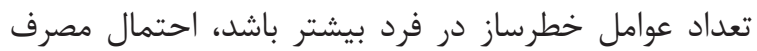

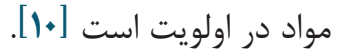

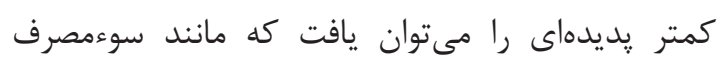

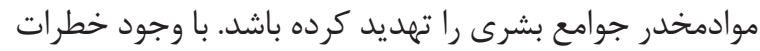

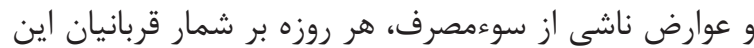

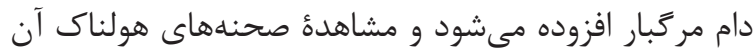

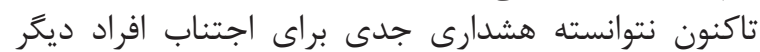

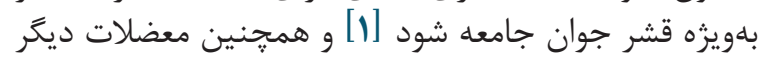

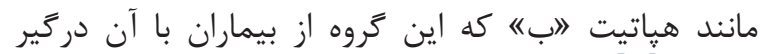

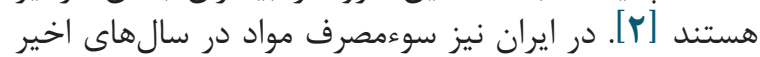

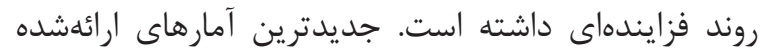

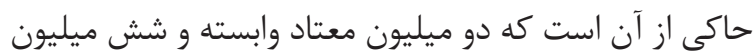

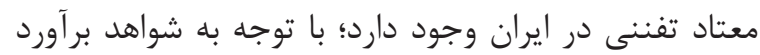

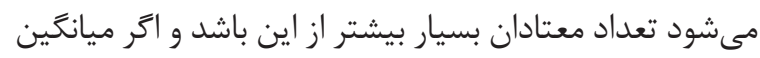

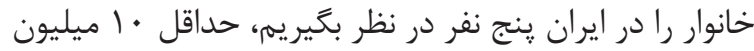

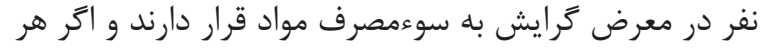

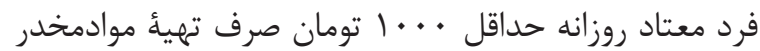

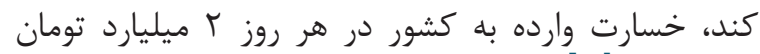

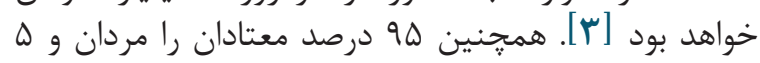




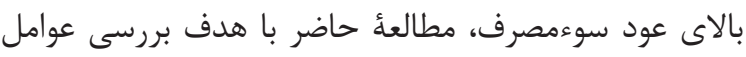

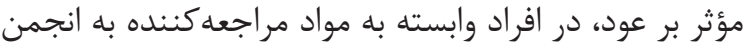

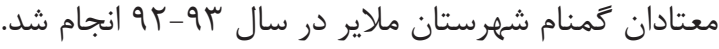

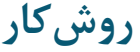

اين يزوهش مطالعهاى توصيفى و از نوع مقطعى (Cross-Sectional)

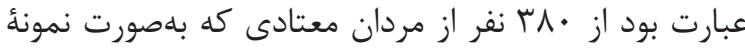

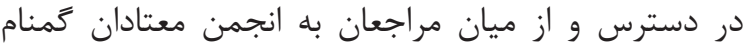

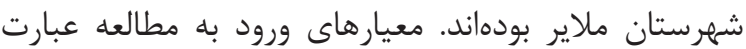

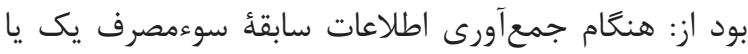

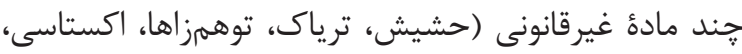

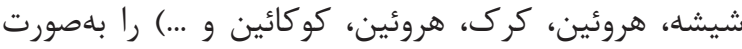

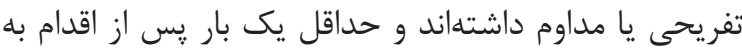

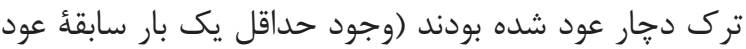

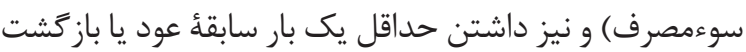

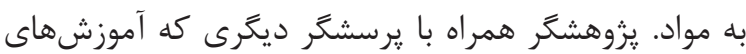

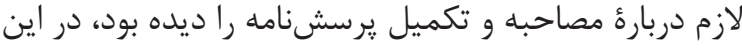

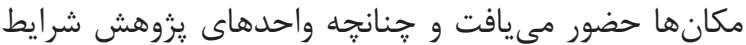

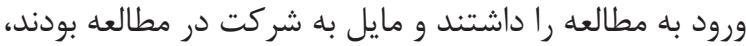

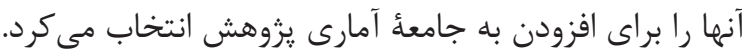

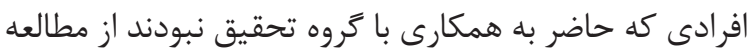
خارج و فرد ديكرى جايكزين آنها مي شئد

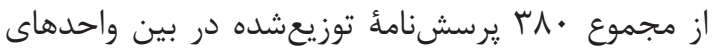

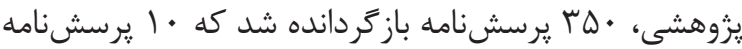

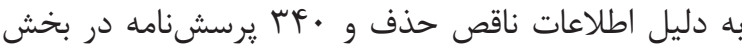
تجزيهوتحليل دادهها به كار كرفته شدف

\section{روش محاسبةٌ حجم نمونه و تعداد آن:}

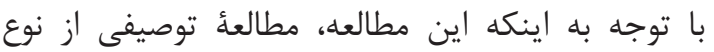

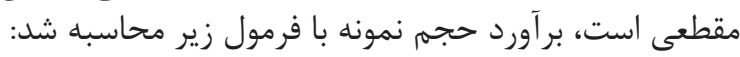

$$
n=\frac{Z_{1-\frac{\alpha}{2}}{ }^{2} p(1-p)}{e^{2}}
$$

با توجه به اينكه در زمان انجام مطالعه ميزان عود در برد بران

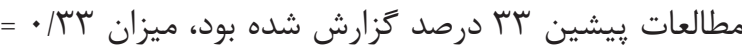

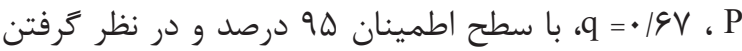

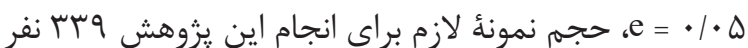

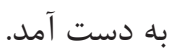

\section{مشخصات ابزار جمع آورى اطلاعات:}

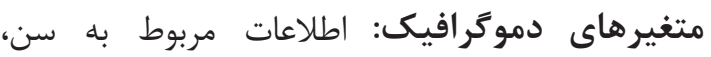

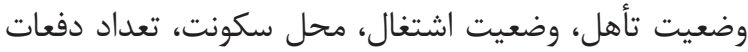

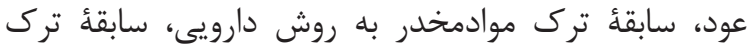
موادمخدر به روش غيردارويى و ... بود.

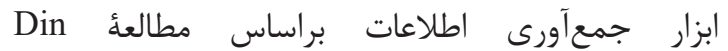
Mohammadi

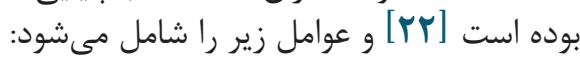

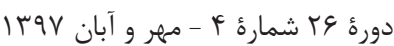

Fridmann

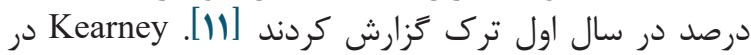

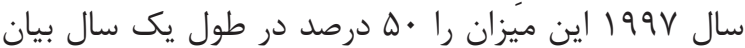

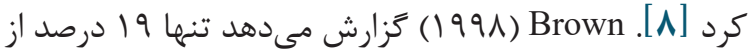

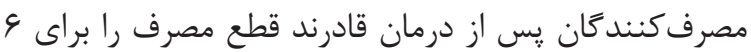

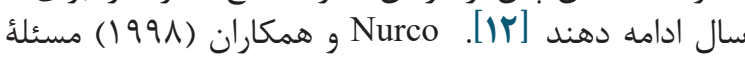

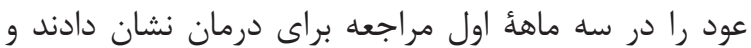

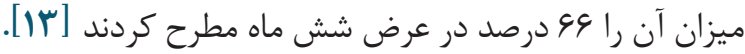
دمان Fadayi

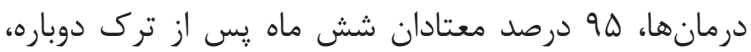

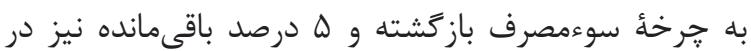

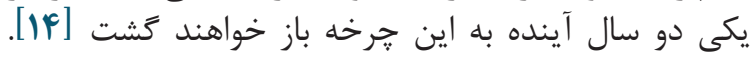

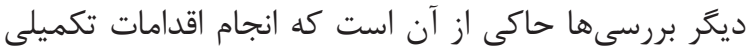

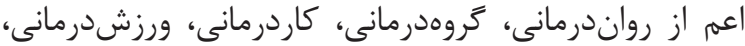

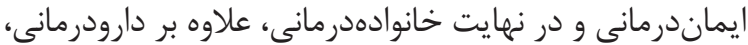

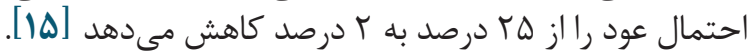

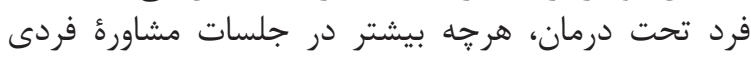

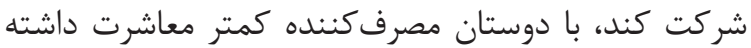

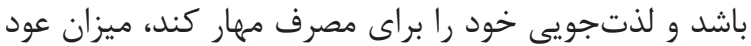

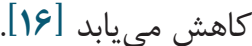

همين طور مطالعات انجامشده روى جانبازان نشان دان داد

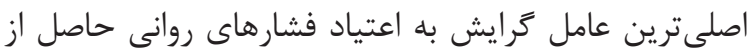

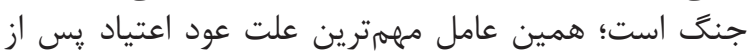

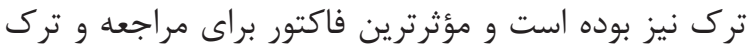

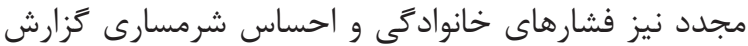

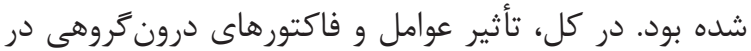

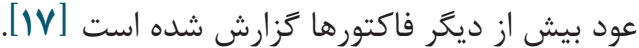

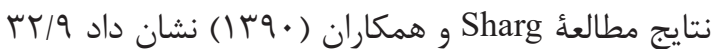

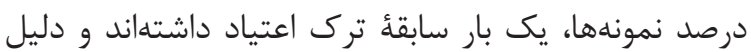

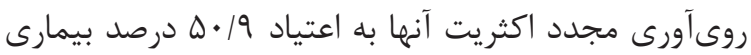

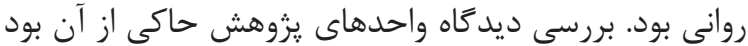

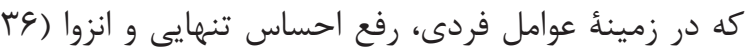

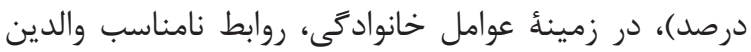

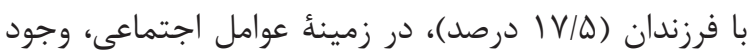

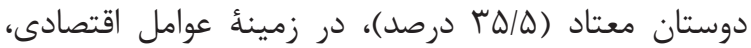

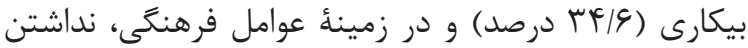

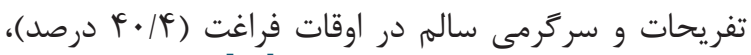

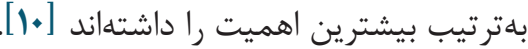
از ديدَاه روانيزشكى و يزشكى، فرد معتاد بيمار است

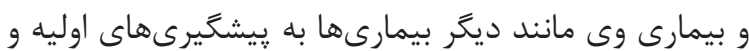
ثانويه نياز دارد [11]].

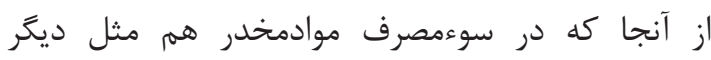

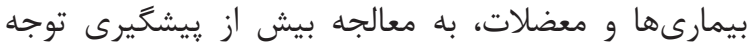

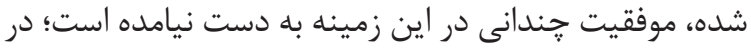

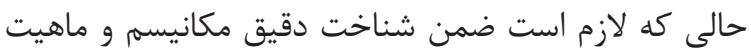

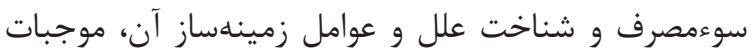
آكاهى و شناخت همكانى نيز فراهم شود. با توجه به آمار 


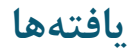

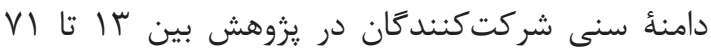

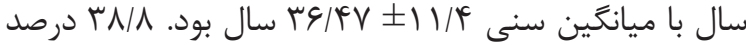

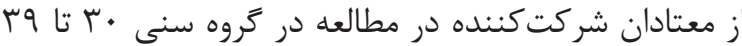

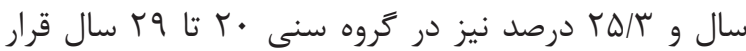

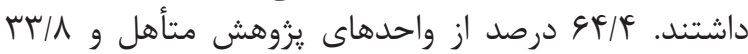

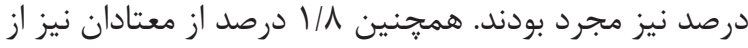

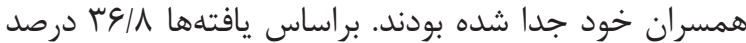

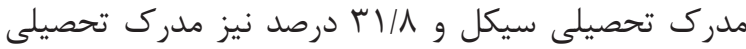

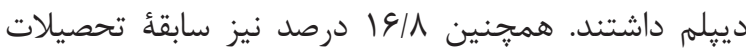

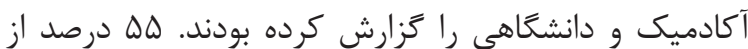

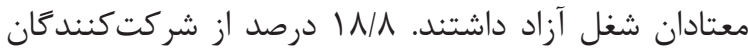

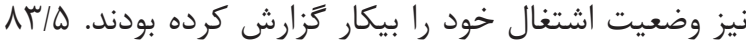

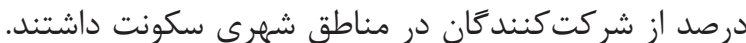

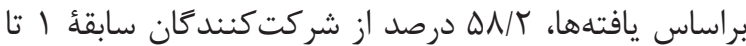

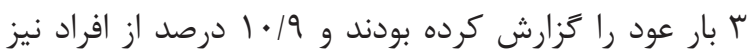
بيش از • ل بار عود داشتهاند.

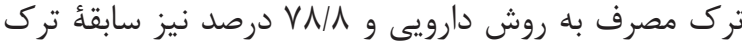

$$
\text { به به روش غيردارويى داشتند. }
$$

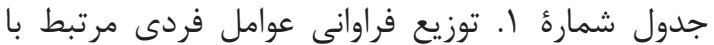

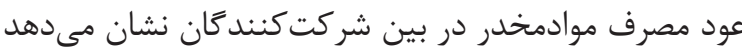

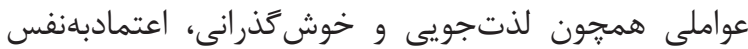

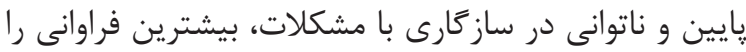

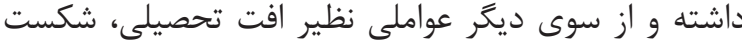

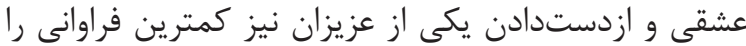

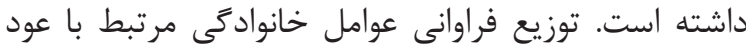

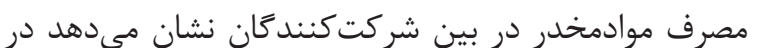

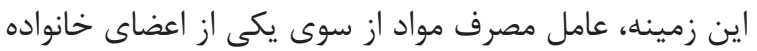

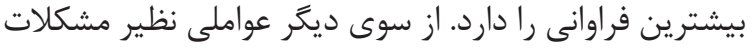

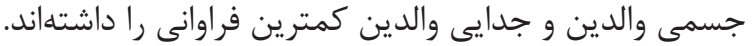
جدول شمارة r. توزيع فراوانى عوامل خانوادگى مربى مربوط

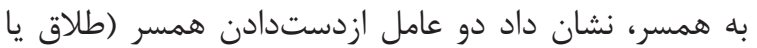

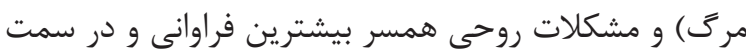

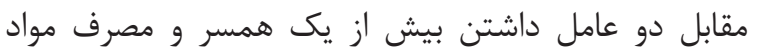

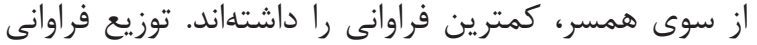

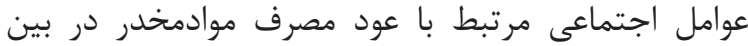

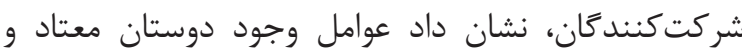

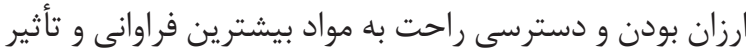

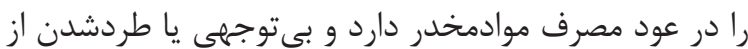

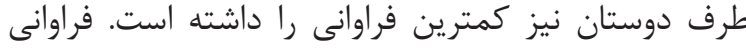

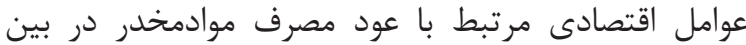

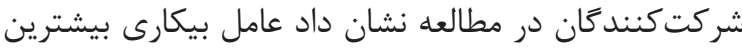

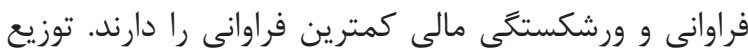

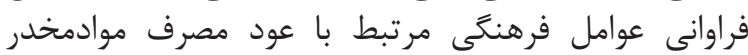

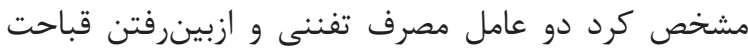

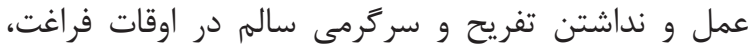

$$
\text { بيشترين فراوانى را داشته است. }
$$

جدول شمارة זّ. ماتريكس همبستخى يِيرسون بين عوامل
يرسشنامة عوامل فردى مرتبط با عود: اين

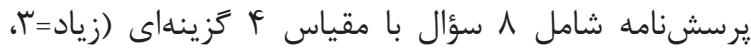

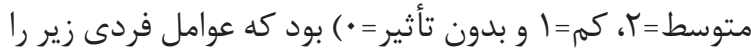

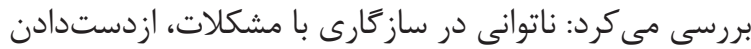

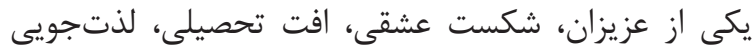

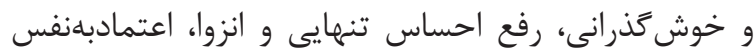

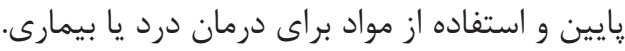

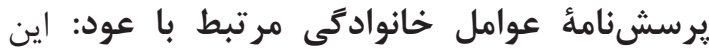

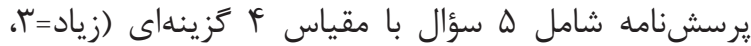

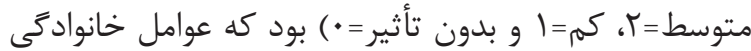

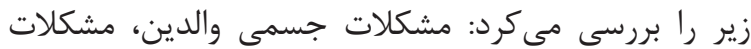

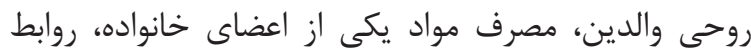
نامناسب بين والدين، جدايى والدين.

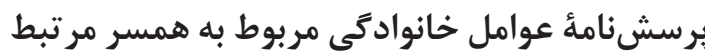

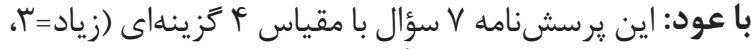

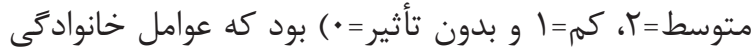

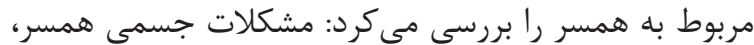

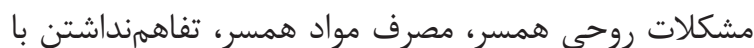

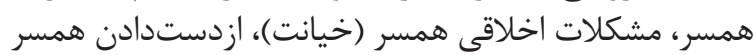
(طلاق يا مرك)، داشتن بيش از يكى همسر (خيان)، ازدر

\section{برسشنامئ عوامل اجتماعى مرتبط با عود: اين}

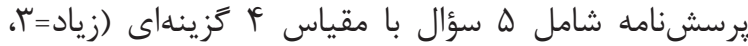

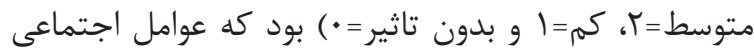

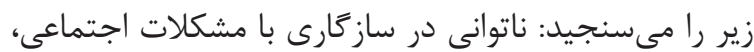

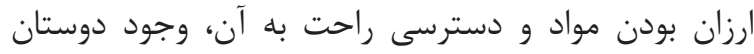

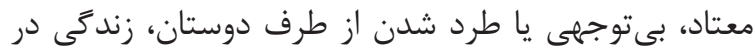
محلههاى شلوغ و يرجمعيت.

\section{يرسشنامة عوامل اقتصادى مرتبط با عود: اين}

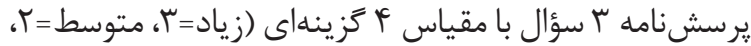

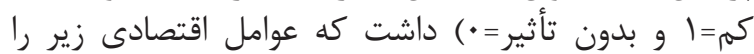

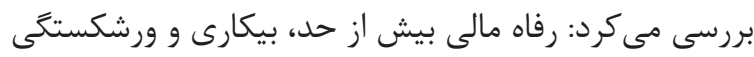

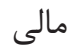

\section{يرسشنامهُ عوامل فرهنكى مرتبط با عود: اين}

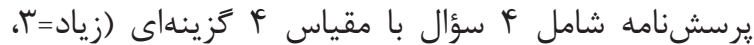

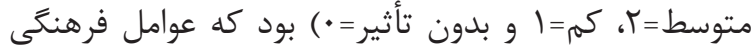

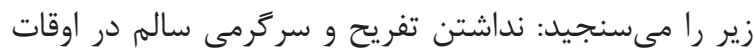

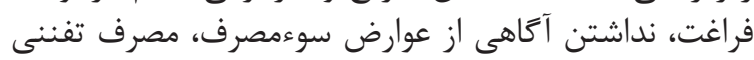

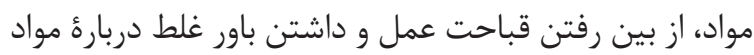

\section{روش تحليل دادهها و آزمون فرضيات}

در اين مطالعه اطلاعات بهدستآمده با نرمافزار آمارى آماري

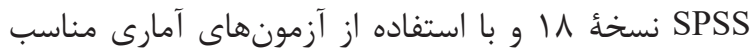

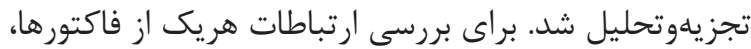

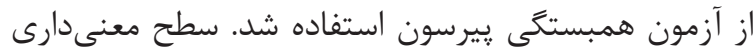
در اين مطالعه هـ/ • در نظر كرفته شد. 


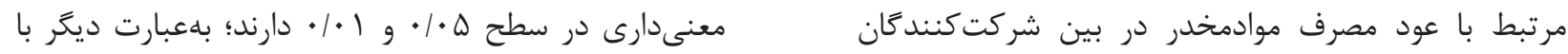

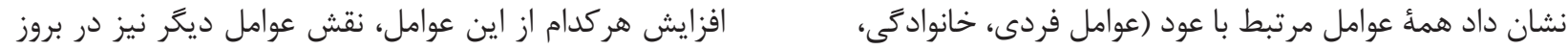

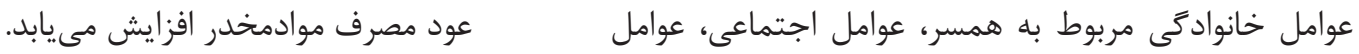

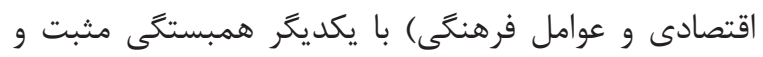

جدول شمارهٔ ا. توزيع فراوانى عوامل فردى و خانوادگى مرتبط با عود مصرف مواد در بين شركتكنندَّان در مطالعه

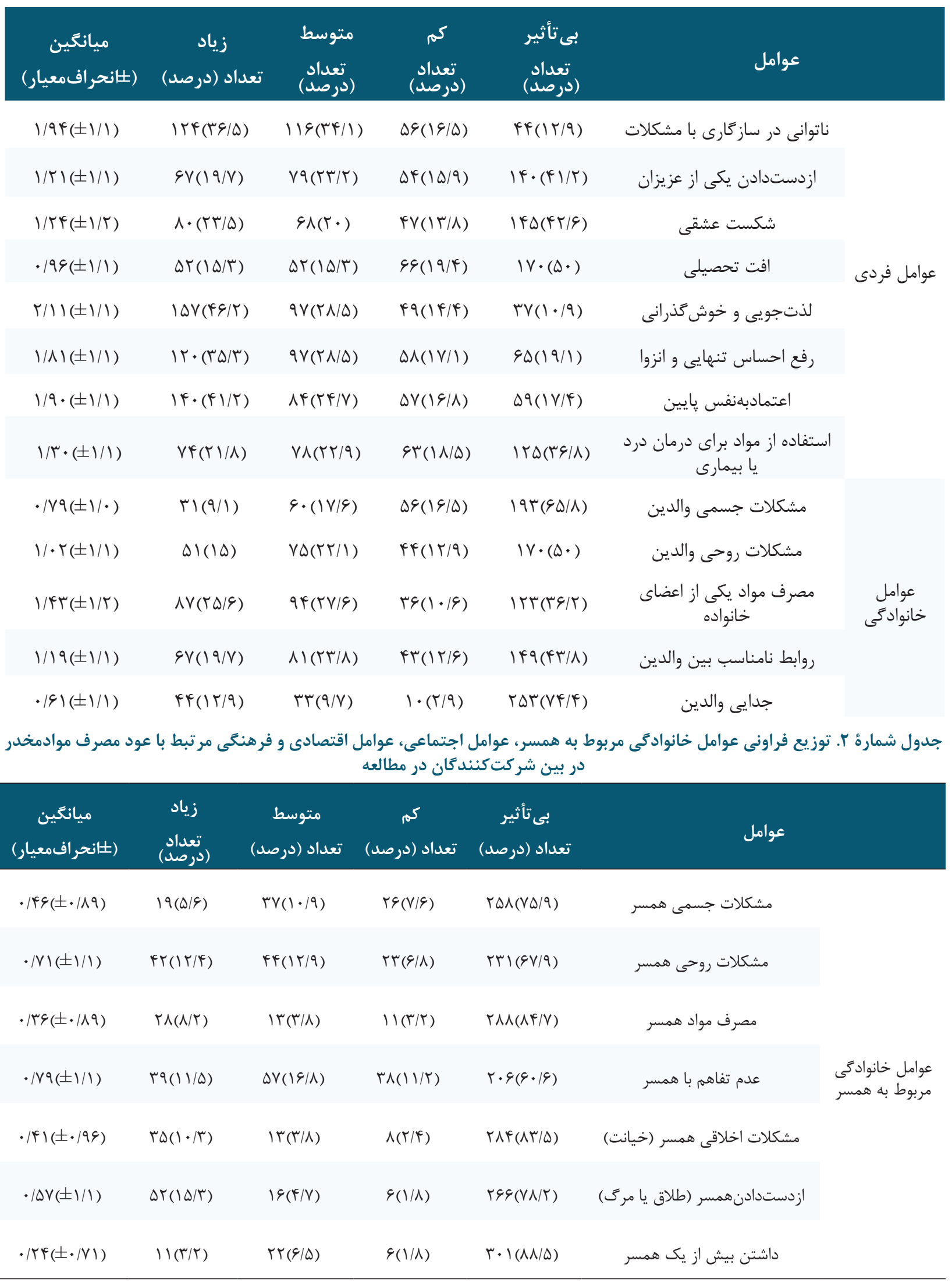




\begin{tabular}{|c|c|c|c|c|c|c|}
\hline 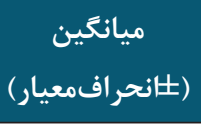 & زياد & 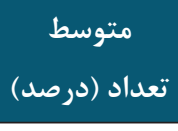 & تعداد (درصد) & تعداد (درصد نأثير & 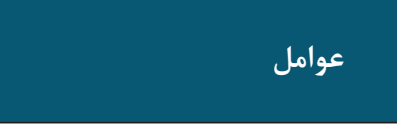 & \\
\hline $1 / 9 \cdot( \pm 1 / 1)$ & $1 \cdot r(r \cdot / r)$ & $\wedge 9(T \& / T)$ & $\Delta V(|\&| \Lambda)$ & $9)(Y \& \mid \wedge)$ & ناتوانى در سازكَارى با مشكلات & \multirow{5}{*}{ عوامل اجتماعى } \\
\hline$r / \cdot \Delta( \pm 1 / 1)$ & $1 \Delta r(F \Delta)$ & $1 \cdot 1(Y 9 / V)$ & $r \Delta(1 \cdot / \mu)$ & $\Delta \backslash(\mid Q)$ & ارزان بودن مواد و دسترسى راحت & \\
\hline$r / I V( \pm 1 / 1)$ & $I V \wedge(\Delta \Gamma / F)$ & $\Lambda M(Y Y / F)$ & $r V(1 \cdot / 9)$ & $\operatorname{Fr}(\mid T / F)$ & وجود دوستان معتاد & \\
\hline $1 / \cdot 9( \pm 1 / 1)$ & $\Delta \Gamma(1 \Delta / \Gamma)$ & $V \backslash(Y \cdot / 9)$ & $V \Psi(Y / \Delta)$ & $\operatorname{IFF}(F T / F)$ & بى بوجهى يا طردشان از طرف & \\
\hline $1 / T F( \pm 1 / 1)$ & $99(\Gamma \cdot / \mu)$ & $V G(Y T / Y)$ & $9)(1 V / 9)$ & $\mid r F(Y q / F)$ & زندگى در محلات شلوغ و يرجمعيت & \\
\hline$\cdot / \wedge \Delta( \pm 1 / 1)$ & $\uparrow 9(\mid r / \Delta)$ & $\Delta r(\mid \Delta / 9)$ & $\lg (\mid r / \Delta)$ & $19 \Delta(\Delta V / Y)$ & رفاه مالى بيش از حد & \multirow{3}{*}{ عوامل اقتصادى } \\
\hline $1 / 99( \pm 1 / 1)$ & $119(Y \mu / 1)$ & $\Lambda Y(Y Y / I)$ & $\Delta r(\mid \Delta / \varphi)$ & $\wedge 9(\Gamma \varphi / T)$ & 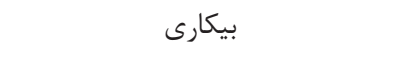 & \\
\hline$\cdot / 9 V( \pm 1 / \pi)$ & $V Y(Y) / D)$ & $\operatorname{Fr}(\mid Y / F)$ & $r \Lambda(\Lambda / r)$ & $19 \vee(\Delta V / 9)$ & ورشكستگى مالى & \\
\hline$r / l f( \pm 1 / 1)$ & $19 \wedge(\uparrow q / 4)$ & $\Lambda \Lambda(Y \Delta / Q)$ & $f q(\mid F / F)$ & $r \Delta(1 \cdot / \mu)$ & نداشتن تفريح و سركرمى سالم در & \multirow{4}{*}{ عوامل اقتصادى } \\
\hline$r / \wedge( \pm 1 / 1)$ & $\mid \Delta \wedge(\uparrow \& \mid \Delta)$ & $q \wedge(\Upsilon \wedge / \wedge)$ & rq(1.19) & $F \wedge(|F /|)$ & نداشتن آكاهى از عوارض سوءمصرف & \\
\hline$\Gamma / \Gamma \varphi( \pm 1 / 1)$ & $1 \wedge \vee(\Delta \Delta)$ & $q \Psi(Y V / F)$ & $r \cdot(\Delta / 9)$ & $F \cdot(1) / \Lambda)$ & مصرف تفننى مواد و از بين رفتن & \\
\hline$r / 1)( \pm 1 / 1)$ & $\mid V F(\Delta \mid / T)$ & $V Y(Y) / T)$ & $\Delta \cdot(I F / V)$ & $\operatorname{Ff}(\mid r / 9)$ & داشتن باور غلط دربارهٔ مواد & \\
\hline
\end{tabular}

جدول شماره ب. ماتريكس همبستخى بين عوامل مرتبط با عود مصرف موادمخدر در بين شركتكنندكًان در مطالعه

\begin{tabular}{|c|c|c|c|c|c|c|}
\hline & & & & & 1 & عوامل فردى \\
\hline & & & & 1 & $\cdot / r q r^{* * *}$ & عوامل خانوادَى \\
\hline & & & 1 & $\left.\cdot|\boldsymbol{f}|\right|^{\text {F*: }}$ & $\cdot /$ MrY**: & عوامل خانوادگى مربوط به همسر \\
\hline & & 1 & $\cdot / T \Delta 9^{* * *}$ & $\cdot / T F D^{* * *}$ & $\cdot / r \Lambda r^{* * *}$ & عوامل اجتماعى \\
\hline & 1 & •/KTY"**: & $\cdot / K r \cdot \cdots$ &.$/ 1 r r^{*}$ & $\cdot / r \Lambda r^{* * *}$ & عوامل اقتصادى \\
\hline 1 & $\cdot / r q \cdot{ }^{* * *}$ & $\cdot / 4 \Lambda \cdot{ }^{* * *}$ & $\cdot / 1 r \cdot *$ & $. / 1 T F=$ & $\cdot / T \Delta \Lambda^{* * *}$ & عوامل فرهنكى \\
\hline
\end{tabular}

مىتواند نقش مهمى در اين مورد داشته باشد. در مطالعه

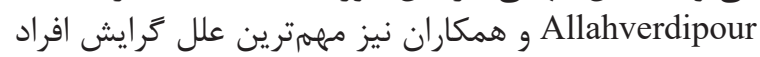

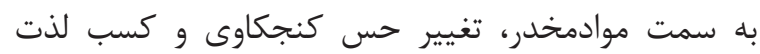

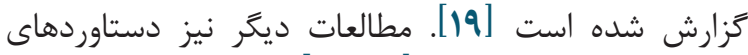

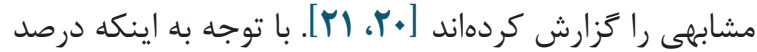

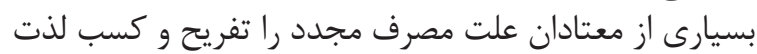

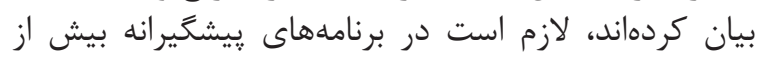
كذشته بر اين عوامل تمركز شود درد
بحث

نتايج مطالعه حاضر در زمينه عوامل فردى مؤثر در در

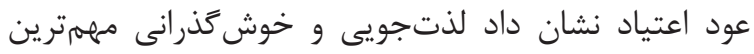

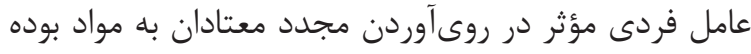

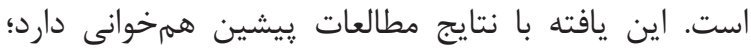

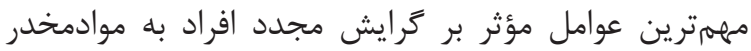

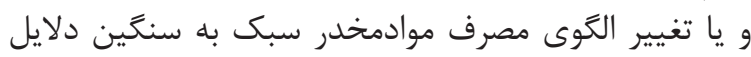

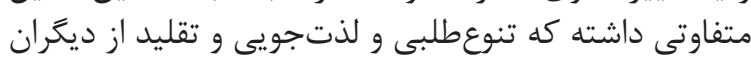




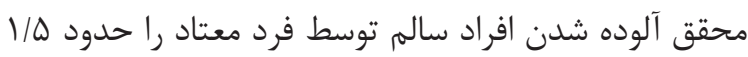

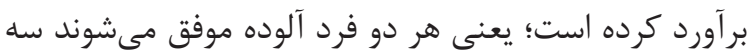
فرد سالم را آلوده كنند؛ بنابر اين آموز

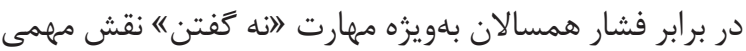

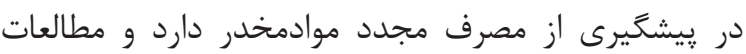

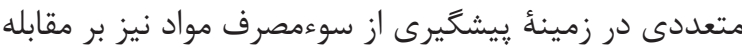

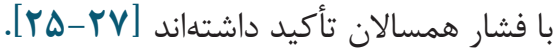

از نظر شركتكنندكان در يزوهش حاضر و در زمينهُ

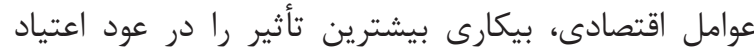

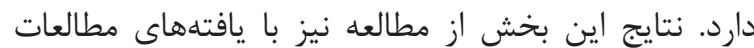

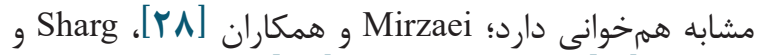

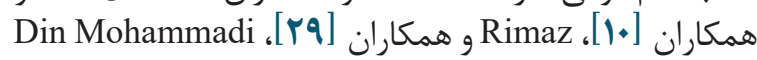

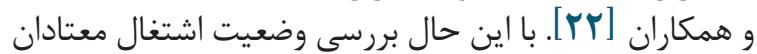

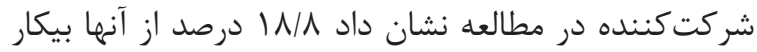

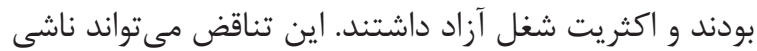

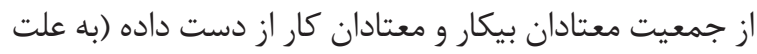

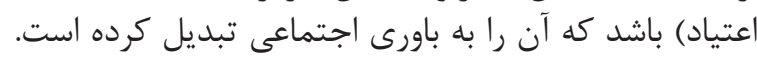

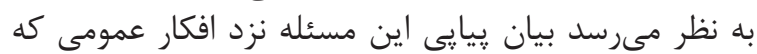

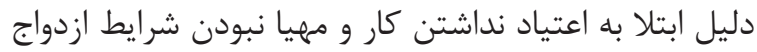

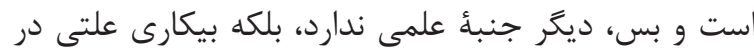
كنار علل ديخر به شمار مىرود.

نتايج مطالعه نشان داد در زمينهُ عوامل فرهنكَى، ازبينرفتن

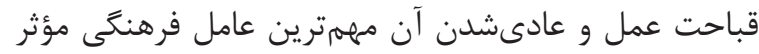

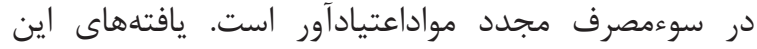

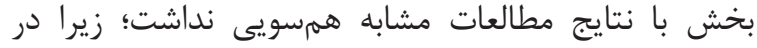

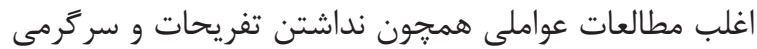

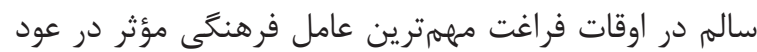

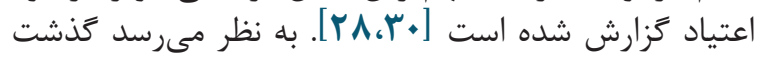

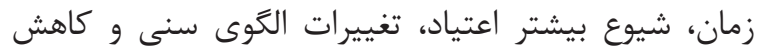

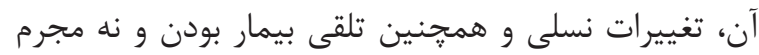

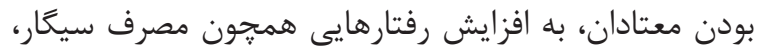

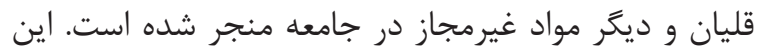

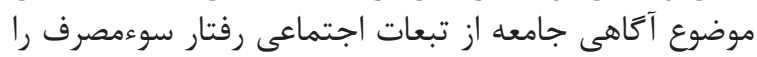

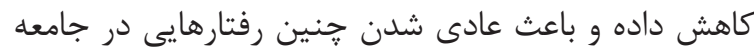

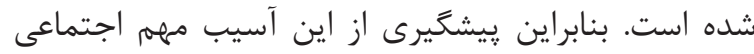

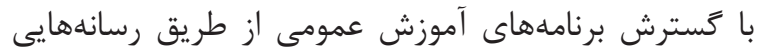

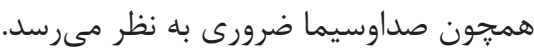

\section{نتيجهَ تيرى}

نتيجأ كلى يزوهش نشان داد در زمينهُ عوامل فردى،

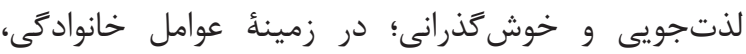

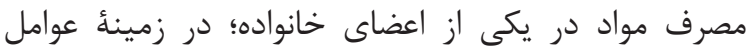

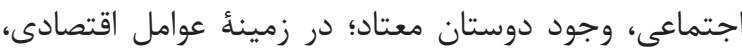

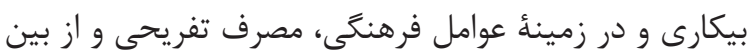

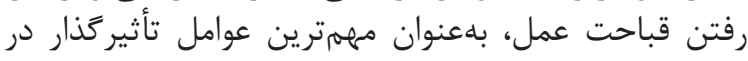
روى آوردن مجدد معتادان به سوءمصن بهرف مونمين مواد است.
نتايج مطالعأُ حاضر در زمينهُ عوامل خانوادكى مؤثر در

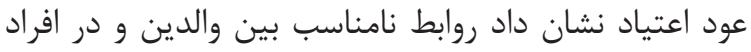

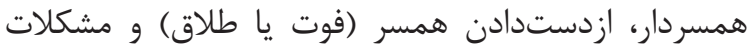

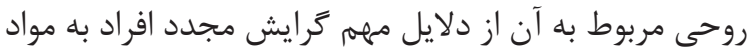

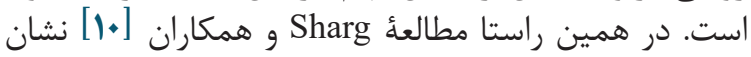

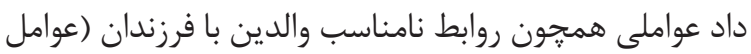

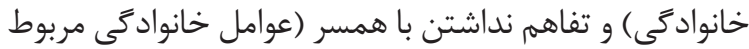

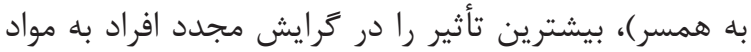

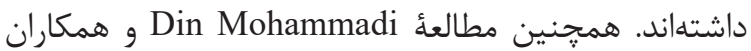

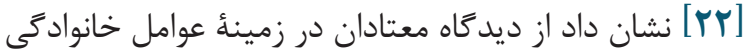

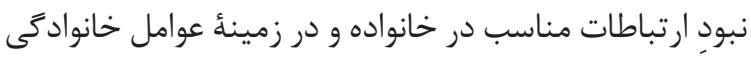

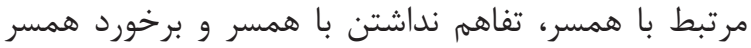

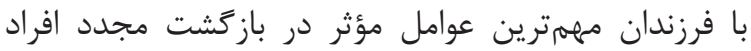

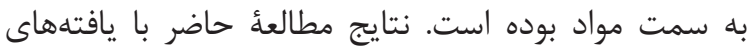
مطالعات مشابه در اين زمينه همخوانى دارد.

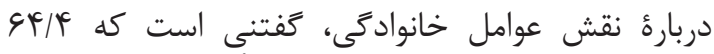

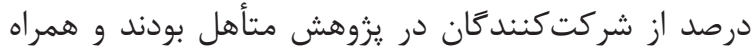

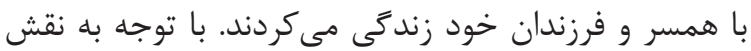

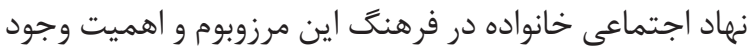

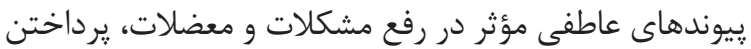

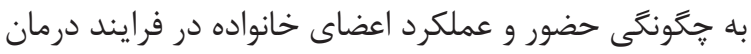

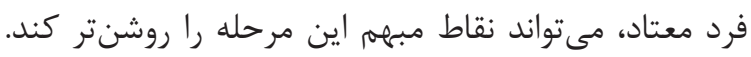

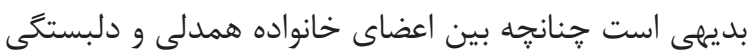

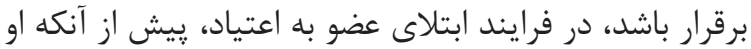

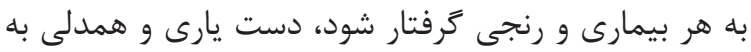

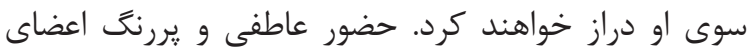

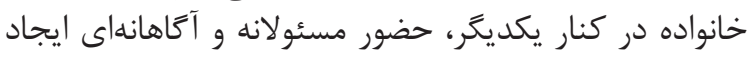

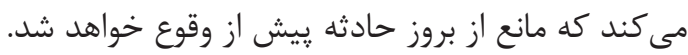

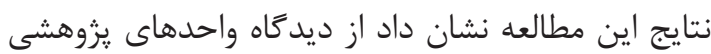

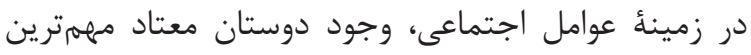

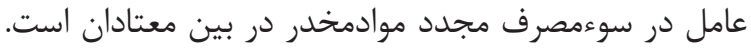

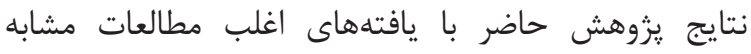

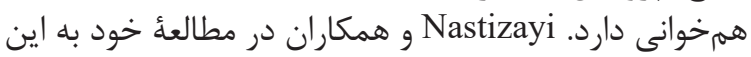

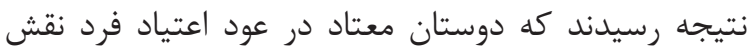

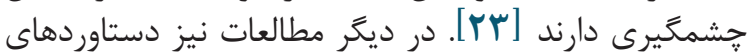

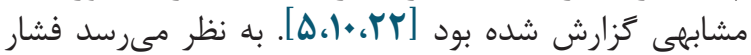

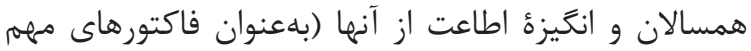

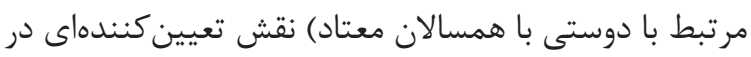

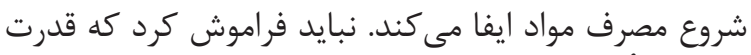

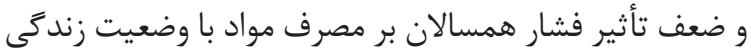

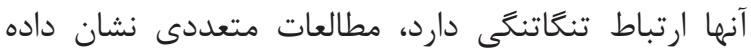

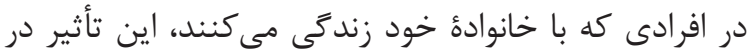

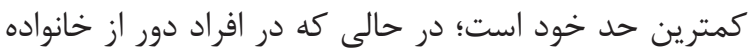

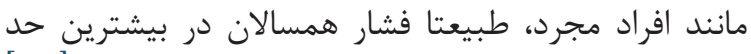

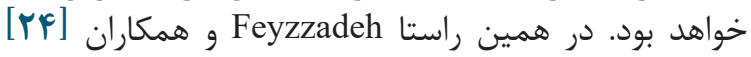

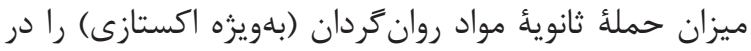

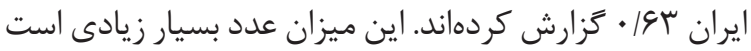

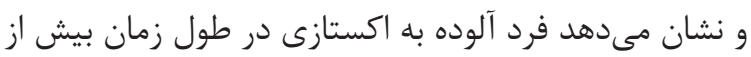

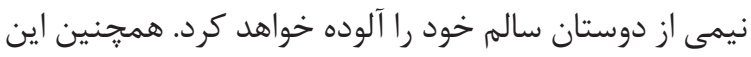




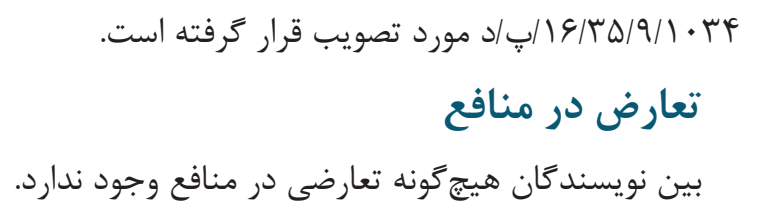

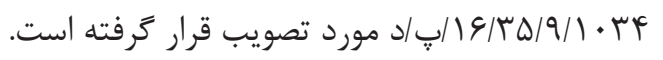

بين نويسندَان هيجَّونه تعارضى در منافع وجود ندارد.

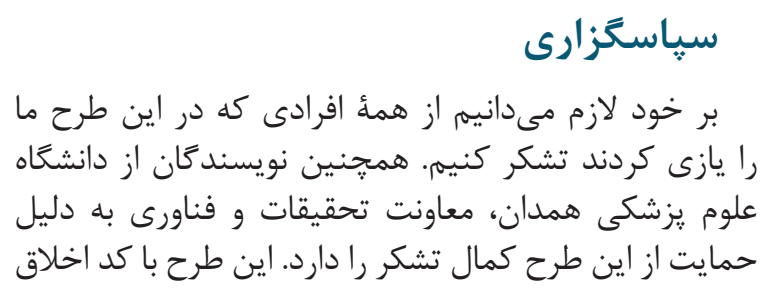

\section{References}

1. Farjad MH. Addiction (Complete and practical manual for families).

2. Khodaveisi M, Salehikha M, Bashirian S, Karami M. Study of preventive behaviors of hepatitis $\mathrm{b}$ based on health belief model among addicts affiliated to Hamedan.

3. Radi M, Rezaei F. Preventive guideline about addiction for families. Sanandaj: Department of Cultural\&Prevention welfare organization of Kurdistan. 2001:1-40.

4. Narenjiha $\mathrm{H}$. The average age of starting drug use is 18 years. Iranian National Drug Control Headquarters. 2008.

5. Sadegiye Ahari S, Azami A, Barak M, Amani F, Firuz S. Reviewing the causes of recurred addiction in patients who referred to centers introduced of Tehran welfare. Ardabil Med Univ J. 2004;3(4):36-40.

6. Sadock BJ, Sadock VA, editors. Kaplan and Sadock's pocket handbook of clinical psychiatry. Lippincott Williams \& Wilkins; 2010.

7. Schaub M, Myslinski E, Schuster C, Krol A, Carbon P. Staf, a promiscuous activator for enhanced transcription by RNA polymerases II and III. EMBO J. 1997;16(1):173-81. PMCid:PMC1169624

8. Kearney MH. Drug treatment for women: Traditional models and new directions. Journal of Obstetric, Gynecologic, \& Neonatal Nursing. 1997;26(4):459-68.

9. Falahzade H, Hoseyni N. Reviewing the causes of recurred addiction from the perspective of addicts who referred to welfare center of Yazd city. Tolooe Behdasht J. 2005;15(1):2.

10. Sharg A, Shakibi A, Neisari R, Aliloo L. Survey of factors related to the relapse of addiction from view of addict patients attending to drug abuse treatment clinics in West Azerbaijan. Urmia Medical Journal. 2011;22(2):129-36.

11. Friedmann PD, Saitz R, Samet JH. Management of adults recovering from alcohol or other drug problems: relapse prevention in primary care. Jama. 1998;279(15):1227-31. https://doi. org/10.1001/jama.279.15.1227 PMID: $\underline{9555766}$
12. Brown BS. Drug Use-Chronic and Relapsing or a Treatable Condition? Substance use \& misuse. 1998;33(12):2515-20. https://doi. org/10.3109/10826089809059338

13. Nurco DN, Kinlock TW, O’Grady KE, Hanlon TE. Differential contributions of family and peer factors to the etiology of narcotic addiction. Drug and Alcohol Dependence. 1998;51(3):229-37. https://doi.org/10.1016/S0376-8716(98)00041-6

14. Fadayi F. percent of addicts after 6 months return to addiction. Iranian National Drug Control Headquarters. 2008.

15. Yegane B. Reducing the risk of recurred addiction from 25 to 2 percent with complementary therapy. Tehran: Iranian Students News Agency. 2007.

16. Hoseini Hajibakandeh A JF, Zar A. Factor related in Relapse. Journal of Social Sciences. 2008;10(41):67-85.

17. Karami G, Ahmadi K, Maleki M, Alipoor M. Addiction relapse in iranian veterans: determination of etiologies and solutions. Iranian Journal of War and Public Health. 2010;2(8):40-4.

18. Yonesi J, Mohammadi M. Using the approach of publishing information on programs to prevent drug addiction among teenagers. Daneshvar Raftar J. 2006;13(16):1-10.

19. Allahverdipour H, Farhadinasab A, Bashirian S, Mahjub H. Pattern of drug abuse among younger adults. Journal of Yazd University of Medical Sciences. 2007;15(4):35-42.

20. Amani F SS, Mohammadi S, Azami A. Pattern of drug use among addiction reffering to drug treatment centers. Journal of Ardabil University of Medical Sciences. 2005;5(3):220-4.

21. Mansouri A R-BM. Prevalence and attitude about using drugs and tobaccos in male students. Zahedan J Res Med Sci (ZJRMS). 2012;13(1):59

22. Din Mohammadi M, Amini K, Yazdan Khah M. Survey of Social and Environmental Factors Related to the Relapse of Addiction in Volunteer Addicted Individuals In Welfare Organization of Zanjan. ZUMS Journal. 2007;15(59):85-94 
23. Nastizayi N, Hezareh Moghadam M, Mollazehi A. The study of factors for recurred addiction from the view of self-introduced addicts to the addiction abandonment centers of zahedan. Journal of Urmia Nursing And Midwifery Faculty. 2010;8(3)

24. Feyzzadeh A MR, Gholestan B. . Ecstasy use velocity among friend of consumers. Hakim. 2004;7(2):1-9

25. Botvin GJ, Griffin KW, Diaz T, Scheier LM, Williams C, Epstein JA. Preventing illicit drug use in adolescents: Long-term follow-up data from a randomized control trial of a school population. Addictive behaviors. 2000;25(5):769-74. https:// doi.org/10.1016/S0306-4603(99)00050-7

26. Swadi H. Individual risk factors for adolescent substance use. Drug and alcohol dependence. 1999;55(3):209-24. $\quad$ https://doi.org/10.1016/ S0376-8716(99)00017-4

27. Hawkins JD, Catalano RF, Miller JY. Risk and protective factors for alcohol and other drug problems in adolescence and early adulthood: implications for substance abuse prevention. Psy- chological bulletin. 1992;112(1):64. https://doi. org/10.1037/0033-2909.112.1.64 PMid: 1529040

28. Mirzaei T, Ravary A, Hanifi N, Miri S, Oskouie F, Mirzaei Khalil Abadi S. Addicts' perspectives about factors associated with substance abuse relapse. Iran Journal of Nursing. 2010;23(67):4958

29. Rimaz S, Dastoorpour M, Mohseni S. Demographic variables associated with relapse in women and men referred to the selected addiction treatment centers in Tehran, 2009: A case-control study. Razi Journal of Medical Sciences. 2013;20(107):63-72

30. Sharg A, Shakibi A, Neisari R, Aliloo L. Survey of factors related to the relapse of addiction from view of addict patients attending to drug abuse treatment clinics in West Azerbaijan. Urmia Medical Journal. 2011;22(2):129-36 
TDS. بررسى عوامل مؤثر بر عود در افراد وابسته به سوءمصرف موادمخدر... 